\title{
A new proof of the bound for the first Dirichlet eigenvalue of the Laplacian operator
}

\author{
Chang-Jun Li and Xiang Gao*
}

\begin{abstract}
In this paper, we present a new proof of the upper and lower bound estimates for the first Dirichlet eigenvalue $\lambda_{1}^{D}(B(p, r))$ of Laplacian operator for the manifold with Ricci curvature $R c \geq-K$, by using Li-Yau's gradient estimate for the heat equation.
\end{abstract}

\section{Introduction and Main Results}

Let $\left(M^{n}, g\right)$ be an $n$-dim $C^{\infty}$ complete Riemannian manifold, and $\Delta$ denote the Laplacian operator. For the compact manifold, it is well known that the eigenvalue problem $-\Delta \varphi=\lambda \varphi$ has discrete eigenvalues, which are listed as

$$
0=\lambda_{0}\left(M^{n}\right)<\lambda_{1}\left(M^{n}\right) \leq \lambda_{2}\left(M^{n}\right) \leq \cdots .
$$

Moreover we call $\lambda_{i}\left(M^{n}\right)$ the $i$ th eigenvalue and call a function $\varphi_{i}$ satisfying $\Delta \varphi_{i}=-\lambda_{i} \varphi_{i}$ the $i$ th eigenfunction.

Recall that the first eigenvalue $\lambda_{1}\left(M^{n}\right)$ for the closed Riemannian manifold $M^{n}$ is defined by

$$
\lambda_{1}\left(M^{n}\right)=\inf _{\phi \in \Omega} \frac{\int_{M^{n}}|\nabla \phi|^{2} d \mu}{\int_{M^{n}} \phi^{2} d \mu},
$$

Key Words: Laplacian operator, Li-Yau gradient estimate, the first Dirichlet eigenvalue. 2010 Mathematics Subject Classification: Primary 53C25; Secondary 53C44.

Received: August, 2012.

Revised: November, 2012.

Accepted: November, 2013.

*The corresponding author: gaoxiangshuli@126.com

This work is supported by the National Natural Science Foundation of China (NSFC) 11301493, 11101267 and Fundamental Research Funds for the Central Universities. 
where $\Omega$ is the completing Hilbert space of

$$
\Omega_{0}=\left\{\phi \in C^{\infty}\left(M^{n}\right) \mid \int_{M^{n}} \phi d \mu=0\right\}
$$

under the norm

$$
\|\phi\|_{1}^{2}=\int_{M^{n}} \phi^{2} d \mu+\int_{M^{n}}|\nabla \phi|^{2} d \mu .
$$

We denote the geodesic ball with center $p$ and radius $r$ by $B(p, r)$ in the $n$-dim manifold $M^{n}$. Then the first Dirichlet eigenvalue $\lambda_{1}^{D}(B(p, r))$ of Laplacian operator can be denoted as

$$
\lambda_{1}^{D}(B(p, r))=\inf _{\phi \in H_{0}^{2}(B(p, r))} \frac{\int_{B(p, r)}|\nabla \phi|^{2} d \mu}{\int_{B(p, r)} \phi^{2} d \mu},
$$

where $H_{0}^{2}(B(p, r))$ is the completing Hilbert space of $C_{0}^{\infty}(B(p, r))$ under the norm

$$
\|\phi\|_{1}^{2}=\int_{B(p, r)} \phi^{2} d \mu+\int_{B(p, r)}|\nabla \phi|^{2} d \mu .
$$

The upper and lower bound for the first eigenvalue of Laplacian operator are very useful in geometry analysis and PDE. In [1], S. Y. Cheng used the approach of Jacobi fields to obtain an upper bound for the first Dirichlet eigenvalue $\lambda_{1}^{D}(B(p, r))$ of Laplacian operator, which are the following comparison theorems.

Theorem 1.1 (Cheng). Let $\left(M^{n}, g\right)$ be a complete Riemannian manifold satisfying $R c \geq(n-1) K$, then

$$
\lambda_{1}^{D}(B(p, r)) \leq \lambda_{1}^{D}\left(B_{K}(p, r)\right),
$$

where $B_{K}(p, r)$ is the geodesic ball in the space form $M_{K}^{n}$, and equality holds iff $B(p, r)$ is isometric to $B_{K}(p, r)$.

Corollary 1.2 (Cheng). Let $\left(M^{n}, g\right)$ be a compact Riemannian manifold satisfying $R c \geq 0$, then

$$
\lambda_{1}\left(M^{n}\right) \leq \lambda_{1}^{D}\left(B_{K}\left(p, \frac{d_{M^{n}}}{2}\right)\right) \leq \frac{C_{n}}{d_{M^{n}}^{2}},
$$

where $C_{n}=2 n(n+4)$ and $d_{M^{n}}$ is the diameter of $M^{n}$.

For the lower bound of the first eigenvalue $\lambda_{1}\left(M^{n}\right)$ for closed Riemannian manifold $M^{n}$, in 1980, P. Li and S. T. Yau [3] used the approach of gradient estimates to derive a lower bound as follows: 
Theorem 1.3 (Li-Yau). Let $\left(M^{n}, g\right)$ be a closed Riemannian manifold satisfying $R c \geq 0$, then

$$
\lambda_{1}\left(M^{n}\right) \geq \frac{\pi^{2}}{2 d_{M^{n}}},
$$

where $d_{M^{n}}$ denotes the diameter of $M^{n}$.

Moreover, their result was improved to a sharp lower bound by J. Q. Zhong and H. C. Yang [5] as follows. Their proofs both rely on the gradient estimate and the maximum principle.

Theorem 1.4 (Zhong-Yang). Let $\left(M^{n}, g\right)$ be a closed Riemannian manifold satisfying $R c \geq 0$, then

$$
\lambda_{1}\left(M^{n}\right) \geq \frac{\pi^{2}}{d_{M^{n}}} .
$$

In this paper, we will present a new and simpler proof of upper and lower bound estimates for the first Dirichlet eigenvalue $\lambda_{1}^{D}(B(p, r))$ of the Laplacian operator for the manifold with Ricci curvature $R c \geq-K$, where $K \geq 0$. Our approach is applying the following gradient estimate which leads to the famous Li-Yau Harnack estimate for the heat equation [4].

Theorem 1.5 (Li-Yau). Let $\left(M^{n}, g\right)$ be a complete Riemannian manifold with Ricci curvature $R c(g) \geq-K$, and let $B(p, 2 r)$ denote the geodesic ball with center $p$ and radius $2 r$ in $M^{n}$. If $u: B(p, 2 r) \times[0, \infty) \longrightarrow \mathbb{R}$ is a positive solution to the heat equation

$$
\frac{\partial}{\partial t} u(x, t)=\Delta u(x, t),
$$

then in $B(p, r)$ we have

$$
\sup _{B(p, r)}\left(\frac{|\nabla u|^{2}}{u^{2}}-\alpha \frac{u_{t}}{u}\right) \leq \frac{C(n) \alpha^{2}}{r^{2}}\left(\frac{\alpha^{2}}{\alpha^{2}-1}+\sqrt{K} r\right)+\frac{n \alpha^{2} K}{2(\alpha-1)}+\frac{n \alpha^{2}}{2 t},
$$

where $\alpha>1$ and $C(n)$ is a constant depended on $n$.

Remark 1. To use Theorem 1.5, we will deal with the solution to the heat equation with the form of $u(x) e^{-\lambda_{1}^{D}(B(p, r)) t}$, where $u(x)$ is the eigenfunction corresponding to the first Dirichlet eigenvalue $\lambda_{1}^{D}(B(p, r))$ of the Laplacian operator, and then apply the Li-Yau's gradient estimate to the solution above and prove an integral estimate as follows. 
Theorem 1.6. Let $\left(M^{n}, g\right)$ be a complete Riemannian manifold with Ricci curvature $R c(g) \geq-K$, then for any geodesic ball $B(p, r)$ in $M^{n}$ and arbitrary $\varepsilon>0$, we have

$$
\begin{aligned}
& \frac{\varepsilon}{t_{2}-t_{1}} \int_{t_{1}}^{t_{2}}|\nabla \log u|^{2} d t \\
\geq & \lambda_{1}^{D}(B(p, r))-\left(\frac{4 C(n) \alpha}{r^{2}}\left(\frac{\alpha^{2}}{\alpha^{2}-1}+\frac{\sqrt{K}}{2} r\right)+\frac{n \alpha K}{2(\alpha-1)}\right)-\frac{n \alpha}{2 t_{1}} \\
& -\frac{1}{4}\left(\alpha+\frac{1}{\varepsilon}\right) \frac{d\left(x_{1}, x_{2}\right)^{2}}{\left(t_{2}-t_{1}\right)^{2}}
\end{aligned}
$$

for arbitrary constant speed minimal geodesic $\gamma(t)$ joining points $x_{1}$ and $x_{2}$ such that $x_{1}, x_{2} \in B\left(p, \frac{r}{2}\right)$ and $x_{1}=\gamma\left(t_{1}\right), x_{2}=\gamma\left(t_{2}\right)$.

By using Theorem 1.6, we can derive the following upper and lower bound estimates for the first Dirichlet eigenvalue $\lambda_{1}^{D}(B(p, r))$ of Laplacian operator.

Theorem 1.7. Let $\left(M^{n}, g\right)$ be a complete Riemannian manifold with Ricci curvature $R c(g) \geq-K$, then for any geodesic ball $B(p, r)$ in $M^{n}$, we have

$$
\frac{C_{4}}{d_{B\left(p, \frac{r}{2}\right)}^{2}}+\frac{C_{2}}{d_{B\left(p, \frac{r}{2}\right)}}+C_{3} \leq \lambda_{1}^{D}(B(p, r)) \leq \frac{C_{1}}{d_{B\left(p, \frac{r}{2}\right)}^{2}}+\frac{C_{2}}{d_{B\left(p, \frac{r}{2}\right)}}+C_{3},
$$

where $d_{B\left(p, \frac{r}{2}\right)}$ denotes the diameter of $B\left(p, \frac{r}{2}\right)$ and $C_{i}(1 \leq i \leq 4)$ are some constants only depending on the choice of $p$ and $r$, which can be seen in the proof.

Remark 2. Recall that for the closed Riemannian manifold, the eigenfunction $u(x)$ corresponding to the first eigenvalue $\lambda_{1}\left(M^{n}\right)$ of the Laplacian operator satisfies

$$
\int_{M^{n}} u(x) d \mu=0
$$

which implies that $u(x)$ can not always be positive. But the Li-Yau's gradient estimate (3) only satisfies for the positive solution of the heat equation, thus in this paper, we deal with the upper and lower bound estimates for the first Dirichlet eigenvalue which can always be positive.

The paper is organized as follows: In section 2, we prove Theorem 1.6 by using the Li-Yau's gradient estimate for heat equations. Based on this, in section 3, we prove Theorem 1.7. 


\section{Proof of Theorem 1.6}

Proof of Theorem 1.6. To prove Theorem 1.6, we consider the function in the form of

$$
\tilde{u}(x, t)=u(x) e^{-\lambda_{1}^{D}(B(p, r)) t},
$$

where $u(x)$ is the eigenfunction corresponding to the first Dirichlet eigenvalue $\lambda_{1}^{D}(B(p, r))$ of the Laplacian operator. It is clear that $\tilde{u}(x, t)$ is actually a solution to the heat equation, in fact

$$
\begin{aligned}
\frac{\partial}{\partial t} \tilde{u}(x, t) & =-\lambda_{1}^{D}(B(p, r)) u(x) e^{-\lambda_{1}^{D}(B(p, r)) t} \\
& =\Delta\left(u(x) e^{-\lambda_{1}^{D}(B(p, r)) t}\right) \\
& =\Delta(\tilde{u}(x, t)) .
\end{aligned}
$$

On the other hand, let $\varphi$ be the eigenfunction corresponding to the first Dirichlet eigenvalue $\lambda_{1}^{D}(B(p, r))$, since $|\nabla| \varphi||=\left|\nabla \sqrt{\varphi^{2}}\right|=\left|\frac{2 \varphi \cdot \nabla \varphi}{2 \sqrt{\varphi^{2}}}\right| \leq|\nabla \varphi|$, we have

$$
\begin{aligned}
\lambda_{1}^{D}(B(p, r)) & =\inf _{\phi \in H_{0}^{2}(B(p, r))} \frac{\int_{B(p, r)}|\nabla \phi|^{2} d \mu}{\int_{B(p, r)} \phi^{2} d \mu} \\
& \leq \frac{\int_{B(p, r)}|\nabla| \varphi||^{2} d \mu}{\int_{B(p, r)}|\varphi|^{2} d \mu} \\
& \leq \frac{\int_{B(p, r)}|\nabla \varphi|^{2} d \mu}{\int_{B(p, r)} \varphi^{2} d \mu} \\
& =\lambda_{1}^{D}(B(p, r)),
\end{aligned}
$$

which implies that $|\varphi|$ is also an eigenfunction corresponding to the first Dirichlet eigenvalue $\lambda_{1}^{D}(B(p, r))$. Thus without loss of generality, we would suppose that the eigenfunction corresponding to the first Dirichlet eigenvalue of Laplacian operator is nonnegative. Then by the strong maximum principle for the Laplacian operator, we can assume that the eigenfunction is positive. Thus $\tilde{u}(x, t)=u(x) e^{-\lambda_{1}^{D}(B(p, r)) t}$ is actually a positive solution to the heat equation.

Then by using the Li-Yau's estimate $(3)$ to $\tilde{u}(x, t)$ we have

$$
\frac{|\nabla \widetilde{u}|^{2}}{\widetilde{u}^{2}}-\alpha \frac{\widetilde{u}_{t}}{\widetilde{u}} \leq \frac{4 C(n) \alpha^{2}}{r^{2}}\left(\frac{\alpha^{2}}{\alpha^{2}-1}+\frac{\sqrt{K}}{2} r\right)+\frac{n \alpha^{2} K}{2(\alpha-1)}+\frac{n \alpha^{2}}{2 t}
$$

satisfies in $B\left(p, \frac{r}{2}\right)$. Then for a constant speed minimal geodesic $\gamma(t)$ joining points $x_{1}$ and $x_{2}$ such that $x_{1}, x_{2} \in B\left(p, \frac{r}{2}\right)$ and $x_{1}=\gamma\left(t_{1}\right), x_{2}=\gamma\left(t_{2}\right)$, we 
have

$$
\begin{aligned}
\log \frac{\tilde{u}\left(x_{2}, t_{2}\right)}{\widetilde{u}\left(x_{1}, t_{1}\right)} & =\int_{t_{1}}^{t_{2}} \frac{d}{d t} \log \tilde{u}(\gamma(t), t) d t \\
& =\int_{t_{1}}^{t_{2}}\left(\frac{\widetilde{u}_{t}}{\widetilde{u}}+\left\langle\frac{\nabla \widetilde{u}}{\widetilde{u}}, \frac{d \gamma}{d t}\right\rangle\right) d t \\
& \geq \int_{t_{1}}^{t_{2}}\left(\frac{\widetilde{u}_{t}}{\widetilde{u}}-\frac{1}{\alpha} \frac{|\nabla \widetilde{u}|^{2}}{\widetilde{u}^{2}}-\frac{\alpha}{4}\left|\frac{d \gamma}{d t}(t)\right|^{2}\right) d t \\
& =-\frac{1}{\alpha} \int_{t_{1}}^{t_{2}}\left(\frac{|\nabla \widetilde{u}|^{2}}{\widetilde{u}^{2}}-\alpha \frac{\widetilde{u}_{t}}{\widetilde{u}}\right) d t-\frac{\alpha}{4} \int_{t_{1}}^{t_{2}}\left|\frac{d \gamma}{d t}(t)\right|^{2} d t \\
& \geq-\int_{t_{1}}^{t_{2}}\left(\frac{4 C(n) \alpha}{r^{2}}\left(\frac{\alpha^{2}}{\alpha^{2}-1}+\frac{\sqrt{K}}{2} r\right)+\frac{n \alpha K}{2(\alpha-1)}+\frac{n \alpha}{2 t}\right) d t \\
& -\frac{\alpha}{4} \int_{t_{1}}^{t_{2}}\left|\frac{d \gamma}{d t}(t)\right|^{2} d t
\end{aligned}
$$

The second inequality we use the Li-Yau's gradient estimate, and it follows from $\gamma(t)$ being a constant speed minimal geodesic that

$$
\left|\frac{d \gamma}{d t}(t)\right| \equiv \frac{d\left(x_{1}, x_{2}\right)}{t_{2}-t_{1}}
$$

Thus we have

$$
\begin{aligned}
& \log u\left(x_{2}\right)-\lambda_{1}^{D}(B(p, r)) t_{2}-\log u\left(x_{1}\right)+\lambda_{1}^{D}(B(p, r)) t_{1} \\
\geq & -\left(\frac{4 C(n) \alpha}{r^{2}}\left(\frac{\alpha^{2}}{\alpha^{2}-1}+\frac{\sqrt{K}}{2} r\right)+\frac{n \alpha K}{2(\alpha-1)}\right)\left(t_{2}-t_{1}\right) \\
& -\frac{n \alpha}{2}\left(\log t_{2}-\log t_{1}\right)-\frac{\alpha}{4} \frac{d\left(x_{1}, x_{2}\right)^{2}}{t_{2}-t_{1}}
\end{aligned}
$$

Then for the path $\gamma(t)$ which is a constant speed minimal geodesic jointing points $x_{1}, x_{2}$ such that $\gamma\left(t_{1}\right)=x_{1}$ and $\gamma\left(t_{2}\right)=x_{2}$ we have

$$
\begin{aligned}
\log u\left(x_{2}\right)-\log u\left(x_{1}\right) & =\log u\left(\gamma\left(t_{2}\right)\right)-\log u\left(\gamma\left(t_{1}\right)\right) \\
& =\int_{t_{1}}^{t_{2}}\left\langle\nabla \log u, \frac{d \gamma}{d t}\right\rangle d t \\
& \leq \int_{t_{1}}^{t_{2}} \varepsilon|\nabla \log u|^{2} d t+\int_{t_{1}}^{t_{2}} \frac{1}{4 \varepsilon}\left|\frac{d \gamma}{d t}\right|^{2} d t \\
& =\int_{t_{1}}^{t_{2}} \varepsilon|\nabla \log u|^{2} d t+\frac{d\left(x_{1}, x_{2}\right)^{2}}{4 \varepsilon\left(t_{2}-t_{1}\right)} .
\end{aligned}
$$


Note that

$$
\log t_{2}-\log t_{1}=\frac{1}{\xi}\left(t_{2}-t_{1}\right) \leq \frac{1}{t_{1}}\left(t_{2}-t_{1}\right),
$$

where $\xi \in\left(t_{1}, t_{2}\right)$. Then by using these two inequalities we have

$$
\begin{aligned}
& \int_{t_{1}}^{t_{2}} \varepsilon|\nabla \log u|^{2} d t+\frac{d\left(x_{1}, x_{2}\right)^{2}}{4 \varepsilon\left(t_{2}-t_{1}\right)}-\lambda_{1}^{D}(B(p, r))\left(t_{2}-t_{1}\right) \\
\geq & -\left(\frac{4 C(n) \alpha}{r^{2}}\left(\frac{\alpha^{2}}{\alpha^{2}-1}+\frac{\sqrt{K}}{2} r\right)+\frac{n \alpha K}{2(\alpha-1)}\right)\left(t_{2}-t_{1}\right)-\frac{n \alpha}{2 t_{1}}\left(t_{2}-t_{1}\right) \\
& -\frac{\alpha}{4} \frac{d\left(x_{1}, x_{2}\right)^{2}}{t_{2}-t_{1}}
\end{aligned}
$$

which implies that

$$
\begin{aligned}
& \frac{\varepsilon}{t_{2}-t_{1}} \int_{t_{1}}^{t_{2}}|\nabla \log u|^{2} d t \\
\geq & \lambda_{1}^{D}(B(p, r))-\left(\frac{4 C(n) \alpha}{r^{2}}\left(\frac{\alpha^{2}}{\alpha^{2}-1}+\frac{\sqrt{K}}{2} r\right)+\frac{n \alpha K}{2(\alpha-1)}\right)-\frac{n \alpha}{2 t_{1}} \\
& -\frac{1}{4}\left(\alpha+\frac{1}{\varepsilon}\right) \frac{d\left(x_{1}, x_{2}\right)^{2}}{\left(t_{2}-t_{1}\right)^{2}}
\end{aligned}
$$

for arbitrary $\varepsilon>0$.

\section{Proof of Theorem 1.7}

In this section, we prove Theorem 1.7 by using Theorem 1.6.

Proof. For the geodesic ball $B\left(p, \frac{r}{2}\right)$, by using the strong maximum principle for the Laplacian operator, we have

$$
u^{2} \geq C_{B\left(p, \frac{r}{2}\right)}\left(\sup _{x \in B\left(p, \frac{r}{2}\right)} u-u\right)\left(u-\inf _{x \in B\left(p, \frac{r}{2}\right)} u\right),
$$

where $C_{B\left(p, \frac{r}{2}\right)}$ is a positive constant only depending on the choice of $p$ and $\frac{r}{2}$.

Then for arbitrary $x, y \in B\left(p, \frac{r}{2}\right)$, we can select the appropriate $s$ and $t$ such that

$$
u(x)=u(\gamma(s))=\inf _{x \in B\left(p, \frac{r}{2}\right)} u
$$


and

$$
u(y)=u(\gamma(t))=\sup _{x \in B\left(p, \frac{r}{2}\right)} u .
$$

Then it follows from (6) that

$$
\begin{aligned}
& \log u(y)-\log u(x) \\
= & \int_{s}^{t}\left\langle\nabla \log u, \frac{d \gamma}{d t}\right\rangle d t \\
\leq & \int_{u(x)}^{u(y)} \frac{d u}{|u|} \\
\leq & \int_{\inf _{x \in B\left(p, \frac{r}{2}\right)} \sup _{x \in B\left(p, \frac{r}{2}\right)^{u}} u}^{u} d u \\
= & \frac{C_{B\left(p, \frac{r}{2}\right)}\left(\sup _{x \in B\left(p, \frac{r}{2}\right)} u-u\right)\left(u-\inf _{x \in B\left(p, \frac{r}{2}\right)} u\right)}{\sqrt{C_{B\left(p, \frac{r}{2}\right)}}} .
\end{aligned}
$$

Then substituting this and (5) into

$$
\begin{aligned}
& \log u\left(x_{2}\right)-\lambda_{1}^{D}(B(p, r)) t_{2}-\log u\left(x_{1}\right)+\lambda_{1}^{D}(B(p, r)) t_{1} \\
\geq & -\left(\frac{4 C(n) \alpha}{r^{2}}\left(\frac{\alpha^{2}}{\alpha^{2}-1}+\frac{\sqrt{K}}{2} r\right)+\frac{n \alpha K}{2(\alpha-1)}\right)\left(t_{2}-t_{1}\right) \\
& -\frac{n \alpha}{2}\left(\log t_{2}-\log t_{1}\right)-\frac{\alpha}{4} \frac{d\left(x_{1}, x_{2}\right)^{2}}{t_{2}-t_{1}}
\end{aligned}
$$

we have

$$
\begin{aligned}
& \lambda_{1}^{D}(B(p, r))\left(t_{2}-t_{1}\right)-\frac{\pi}{\sqrt{C_{B\left(p, \frac{r}{2}\right)}}}-\frac{n \alpha}{2 t_{1}}\left(t_{2}-t_{1}\right) \\
& \leq\left(\frac{4 C(n) \alpha}{d_{B\left(p, \frac{r}{2}\right)}^{2}}\left(\frac{\alpha^{2}}{\alpha^{2}-1}+\frac{\sqrt{K}}{2} d_{B\left(p, \frac{r}{2}\right)}\right)+\frac{n \alpha K}{2(\alpha-1)}\right)\left(t_{2}-t_{1}\right) \\
& +\frac{\alpha}{4} \frac{d_{B\left(p, \frac{r}{2}\right)}^{2}}{t_{2}-t_{1}}
\end{aligned}
$$

where $d_{B\left(p, \frac{r}{2}\right)}$ denotes the diameter of $B\left(p, \frac{r}{2}\right)$. This implies that 


$$
\begin{aligned}
& \lambda_{1}^{D}(B(p, r)) \\
\leq & \left(\frac{4 C(n) \alpha}{d_{B\left(p, \frac{r}{2}\right)}^{2}}\left(\frac{\alpha^{2}}{\alpha^{2}-1}+\frac{\sqrt{K}}{2} d_{B\left(p, \frac{r}{2}\right)}\right)+\frac{n \alpha K}{2(\alpha-1)}\right)+\frac{n \alpha}{2 t_{1}} \\
& +\frac{\pi}{\sqrt{C_{B\left(p, \frac{r}{2}\right)}}\left(t_{2}-t_{1}\right)}+\frac{\alpha}{4} \frac{d_{B\left(p, \frac{r}{2}\right)}^{2}}{\left(t_{2}-t_{1}\right)^{2}}
\end{aligned}
$$

for any $t_{1}<t_{2}$, and

$$
\begin{aligned}
& \lambda_{1}^{D}(B(p, r)) \\
\geq & \left(\frac{4 C(n) \alpha}{d_{B\left(p, \frac{r}{2}\right)}^{2}}\left(\frac{\alpha^{2}}{\alpha^{2}-1}+\frac{\sqrt{K}}{2} d_{B\left(p, \frac{r}{2}\right)}\right)+\frac{n \alpha K}{2(\alpha-1)}\right)+\frac{n \alpha}{2 t_{1}} \\
& -\frac{\pi}{\sqrt{C_{B\left(p, \frac{r}{2}\right)}}\left(t_{1}-t_{2}\right)}+\frac{\alpha}{4} \frac{d_{B\left(p, \frac{r}{2}\right)}^{2}}{\left(t_{1}-t_{2}\right)^{2}}
\end{aligned}
$$

for any $t_{1}>t_{2}$.

Furthermore, by selecting the parameters

$$
t_{1}=d_{B\left(p, \frac{r}{2}\right)}^{2}
$$

and

$$
t_{2}=\left(1+\frac{1}{\sqrt{C_{B\left(p, \frac{r}{2}\right)}}}\right) d_{B\left(p, \frac{r}{2}\right)}^{2},
$$

and substituting them into above, we have

$$
\begin{aligned}
& \lambda_{1}^{D}(B(p, r)) \\
\leq & \left(\frac{4 C(n) \alpha}{d_{B\left(p, \frac{r}{2}\right)}^{2}}\left(\frac{\alpha^{2}}{\alpha^{2}-1}+\frac{\sqrt{K}}{2} d_{B\left(p, \frac{r}{2}\right)}\right)+\frac{n \alpha K}{2(\alpha-1)}\right)+\frac{n \alpha}{2 d_{B\left(p, \frac{r}{2}\right)}^{2}} \\
& +\frac{\pi}{d_{B\left(p, \frac{r}{2}\right)}^{2}}+\frac{\alpha C_{B\left(p, \frac{r}{2}\right)}}{4 d_{B\left(p, \frac{r}{2}\right)}^{2}} \\
= & \frac{C_{1}}{d_{B\left(p, \frac{r}{2}\right)}^{2}}+\frac{C_{2}}{d_{B\left(p, \frac{r}{2}\right)}}+C_{3} .
\end{aligned}
$$


For the same reason, we can substitute the parameters

$$
t_{1}=\left(1+\frac{1}{\sqrt{C_{B\left(p, \frac{r}{2}\right)}}}\right) d_{B\left(p, \frac{r}{2}\right)}^{2}
$$

and

$$
t_{2}=d_{B\left(p, \frac{r}{2}\right)}^{2}
$$

into the same inequality to obtain that

$$
\begin{aligned}
& \lambda_{1}^{D}(B(p, r)) \\
\geq & \left(\frac{4 C(n) \alpha}{d_{B\left(p, \frac{r}{2}\right)}^{2}}\left(\frac{\alpha^{2}}{\alpha^{2}-1}+\frac{\sqrt{K}}{2} d_{B\left(p, \frac{r}{2}\right)}\right)+\frac{n \alpha K}{2(\alpha-1)}\right) \\
& +\frac{n \alpha \sqrt{C_{B\left(p, \frac{r}{2}\right)}}}{2\left(1+\sqrt{\left.C_{B\left(p, \frac{r}{2}\right)}\right)} d_{B\left(p, \frac{r}{2}\right)}^{2}\right.}-\frac{\pi}{d_{B\left(p, \frac{r}{2}\right)}^{2}}+\frac{\alpha C_{B\left(p, \frac{r}{2}\right)}}{4 d_{B\left(p, \frac{r}{2}\right)}^{2}} \\
= & \frac{C_{4}}{d_{B\left(p, \frac{r}{2}\right)}^{2}}+\frac{C_{2}}{d_{B\left(p, \frac{r}{2}\right)}}+C_{3} .
\end{aligned}
$$

\section{References}

[1] S. Y. Cheng, Eigenvalue comparison and its geometric application, Math. Z. 143 (1975), 289-297.

[2] B. Chow, P. Lu, L. Ni, Hamilton's Ricci Flow. Lectures in Contemporary Mathematics, 3, Science Press and Graduate Studies in Mathematics, 77, American Mathematical Society (co-publication), 2006.

[3] P. Li and S. T. Yau, Eigenvalues of a compact Riemannian manifold, AMS Proc. Symp. Pure Math. 36 (1980), 205-239.

[4] R. Schoen and S. T. Yau, Lectures on differential geometry. Conference Proceedings and Lecture Notes in Geometry and Topology, I. International Press, Cambridge, MA, 1994.

[5] J. Q. Zhong and H. C. Yang, On the estimate of first eigenvalue of a compact Riemannian manifold, Sci. Sinica Ser. A 27 (1984), 1265-1273. 
Chang-Jun Li,

School of Mathematical Sciences,

Ocean University of China,

Lane 238, Songling Road, Laoshan District, Qingdao City,

Shandong Province, 266100, People's Republic of China.

Email: licj@ouc.edu.cn

Xiang Gao,

School of Mathematical Sciences,

Ocean University of China,

Lane 238, Songling Road, Laoshan District, Qingdao City,

Shandong Province, 266100, People's Republic of China.

Email: gaoxiangshuli@126.com 
